\title{
@@- OPENACCESS EXPLORING CAUSES OF STILLBIRTHS THROUGH RECODE check for updates CLASSIFICATION: A 4 YEAR EXPERIENCE IN TERTIARY CARE HOSPITAL
}

Department of Obstetrics \& Gynecology, Lady Reading Hospital (Medical Teaching Hospital), Peshawar-Pakistan

Address for correspondence: Nazish Hayat

Department of Obstretic \& Gynaecology, Lady Reading Hospital (Medical Teaching Hospital), Peshawar-Pakistan

E-mail:

nazish_2110@yahoo.com

Date Received:

September, $18^{\text {th }} 2020$

Date Revised:

December, $27^{\text {th }} 2021$

Date Accepted:

December, $27^{\text {th }} 2021$

Nazish Hayat ${ }^{\star}$, Laila Zeb, Mehtab Shah

ABSTRACT

Objective: To study the causes of stillbirths through ReCoDe classification over 4 years in tertiary care hospital.

Methodology: This descriptive study, with non-probability consecutive sampling was carried out from January 2016 till December 2019 in Department of Obstetrics \& Gynecology, Lady Reading Hospital (Medical Teaching Hospital), Peshawar-Pakistan. After taking the ethical approval, relevant parameters at death were recorded against the headings of ReCoDe classification (along with the sub classifications) as a. Fetus b. Umbilical cord c. Placenta d. Amniotic fluid e. Uterus f. Mother g. Intrapartum h. Trauma i. Unclassified. Data was analyzed on the SPSS v.23.0

Results: The stillbirth rate recorded over 4 studied years was 7.8\%, 6.4\%, 5.9\%and 4.7\% in $2016,2017,2018$ and 2019 respectively. It was observed that the most common relevant conditions at birth of stillborn babies were associated with fetal, maternal and placental causes. Fetal causes were, however, mainly contributed by congenital abnormalities $(65.63 \%, 79 \%, 58.11 \%$ and $74 \%$ respectively). Among the placental causes, placental abruption remained the main killer $(54 \%, 72 \%, 78 \%$ and $76 \%)$. Among the maternal causes, diabetes and hypertension contributed the most. Of other notable causes, ruptured uterus was found to be an important entity taking its toll each year (6\%, 4\%, 6\% and 5.3\% respectively).

Conclusion: The study concluded that overall placental causes were more pre-dominant cause for still births followed by fetal causes. Placental abruption, and congenital anomaly were the most common sub-causes among their categories of placental and fetal causes respectively.

Key Words: Stillbirths; ReCoDe classification; abruption; congenital abnormalities.

\section{INTRODUCTION}

Still birth remains a tragedy that impacts the lives of many. It is the birth of a fetus after having reached the age of viability, with no signs of life at birth. Since in present day and age, many of the unwanted pregnancies are dealt with before they reach the age of viability, these are mainly the wanted pregnancies that face the unfortunate fate. Globally, about 1.9 million stillbirth occur annually. ${ }^{1}$ The distribution of still births burden population-wise is, however, not uniform, and this relates mainly, to the standard of healthcare services provided in different parts of the world. This in turn, reflects the resources of each country and the socioeconomic conditions prevalent therein. The main brunt of the situation is borne by low resourced countries as WHO reports that $98 \%$ of all stillbirths occur in low and middle income countries as against only $2 \%$ in developed nations. ${ }^{2}$ Even more alarming is the fact, that many cases of stillbirths occurring in low re- sourced countries are not even recorded. This implies that the situation is even worse than said. On the other hand, in developed countries, the rate at which viable fetuses are lost before they enter the world, has been brought down significantly over the years. The current quoted figures for stillbirth rate is $3 / 1000$ births in the UK, 2.7/1000 births in Canada and 2/1000 births in Finland. ${ }^{3}$ This has to be viewed in light of the fact that these countries have better and efficient reporting systems in place, ensuring that each stillbirth gets counted. Like all other diseases and medical conditions, the prevalence of different etiological entities for stillbirths is different in different populations. This means that the first and foremost step in any attempt to reduce the incidence of stillbirth in a country is to identify the causes and risk factors. It is only after this, that a focused and targeted approach to tackle it can bring about any improvement.

Predictably, stillbirth rate in Pakistan is no better 
than other low resourced countries. UNICEF database shows a figure in the range of 30 $37 / 1000$ births over the past decade ${ }^{3}$, rate of stillbirths in Pakistan as high as 53.5/1000 livebirths in the period between 2010 till 2018 has been quoted, as against the estimate of stillbirth rate of 23.2/1000 livebirths in other low and middle income countries. ${ }^{4}$ However, it can be rightly assumed that this reported figure is just the tip of the iceberg, keeping in mind the lack of stillbirth reporting and record keeping at local as well as national level. Even where it is recorded, there is lack of proper classification system, leading to ineffective data keeping. This implies that there is a lack of basic information to start with, if any successful effort to bring down the stillbirth rate in Pakistan has to be made.

Not very long ago, in the year 2000, 189 heads of states, including Pakistan, in the UN, signed the time-bound developmental pact, The Millenium Declaration, to combat extreme poverty and provision of basic human rights of health, education and security. ${ }^{5}$ It did not address stillbirths directly but aimed to increase the number of births attended by skilled birth attendants, which in practice, could bring about some degree of reduction in stillbirth rate. However it was clearly recognized when progress was assessed over years that Pakistan lacked the civil registry system for data recording. ${ }^{6}$

Although not specifically covered in MilIennium Development Goals, the world however turned its attention towards stillbirths over time. Every Newborn Action Plan, was set up in 2014, led by UNICEF and WHO.? It presents evidence-based solutions to prevent newborn deaths and stillbirth. Lessons from maternal and newborn mortality suggest the need for agreement on credible measures of stillbirth burden and causes, a classification of stillbirth to present them, and evidence for simple and cost-effective interventions with indicators for monitoring. 8,9 Throughout the developed regions of the world, healthcare systems are using various classification systems for recording stillbirths. These include ReCoDe (relevant condition at death), PSANZ-PDC, Tulip and CODAC. Aim of recording this data is not only to document different causes of stillbirths, but also to identify areas where productive efforts can be made in healthcare systems to bring down stillbirth rate, in the context of available resources, cost-effectively by bringing a change in modifiable risk factors.

In our study we have tried to collect and organize the data related to the causes of stillbirths in patients presenting to tertiary care hospital, in light of ReCoDe classification system with an aim to identify areas where cost-effective strategies can be designed to bring improvement in this neglected area of healthcare.

\section{METHODOLOGY}

This descriptive study, with non-probability consecutive sampling was conducted at Department of Gynaecology and Obstetrics, Lady Reading Hospital (Medical Teaching Institute), Peshawar. Approval was taken from the ethical committee of the hospital and data was collected over a period of 4 years stating from $1^{\text {st }}$ January 2016 till $31^{\text {st }}$ December 2019. Stillbirth was defined as birth of a baby with no signs of life, at or after 28 completed weeks of gestation, the gestational age cut off being set the same as is being used in international comparison studies e.g. by UN IGME'1. All pregnant women diagnosed as having dead fetus after 28 weeks of gestation, which corresponded to late fetal death in $10^{\text {th }}$ and $11^{\text {th }}$ revision of $I C D^{10}$ were included in the study. Those patients who had intrapartum fetal demise leading to stillbirth were also included in the study. All pregnant women who had live birth were excluded from the study. Dead fetuses born prior to 28 weeks of gestation were also excluded from the study. After brief demographic data, relevant condition at death was noted and cause of death recorded as was deemed most probable in individual cases. However, no autopsy was performed. Data was recorded against ReCoDe classification as a. FETUS (Lethal congenital abnormality, Infection, Non-immune hydrops, Iso-immunisation, Fetomaternal Hamorrhage evaluated by performing acid elution (KB) test, Meconium aspiration, IUGR), b. UMBILICAL CORD (Prolapse, Loopslknots, Velamentous insertion, Unknown), c. PLACENTA (Abruption, Previa, Vasa previa, Placental insufficency/ infarct, Unclassified APH), d. AMNIOTIC FLUID (Chorioamnionitis, Oligohydramnios, Polyhydramnios) e. UTERUS (Rupture, Others) f. MOTHER (Diabetes, Thyroid, Essential HTN, Hypertensive disorders of pregnancy, Lupus/ APLS, Cholestasis, Drug Abuse, Obstructed labor, HELLP) g. INTRAPARTUM (Asphyxia, Birth Trauma) h. TRAUMA (External, latrogenic) i. UNCLASSIFIED (No relevant condition identified, No information available). Data was analysed on the Statistical Package for Social Science (SPSS) version 23. Continuous data were showed as the mean \pm standard deviation, while the categorical and nominal data were presented as frequencies and percentages.

\section{RESULTS}

The stillbirth rate recorded over 4 studied years was $7.8 \%, 6.4 \%, 5.9 \%$ and $4.7 \%$ in 2016, 2017, 2018 and 2019 respectively, the average rate being $6.2 \%$. In the year prior to specify 4 years, i.e. during 2015 , the figure was $9.4 \%$ when the stillbirths were not recorded according to RECODE classification. It was observed that the most common relevant conditions at birth of stillborn babies were associated with fetal, maternal and placental causes (table 1). Fetal causes made up $19.2 \%$ on the average. When studied individually, congenital abnormalities were found to be mainly responsible (65.63\%, 79\%, 58.11\% and 74\%), making up about $14 \%$ of all stillbirths. Among the placental causes, that were responsible for 
Table 1: Causes for the still birth

\begin{tabular}{|c|c|c|c|c|c|}
\hline Causes & 2016 & 2017 & 2018 & 2019 & Average \\
\hline Fetus & $16.24 \%$ & $24.20 \%$ & $14.00 \%$ & $22.50 \%$ & $19.20 \%$ \\
\hline Placenta & $36.04 \%$ & $23.94 \%$ & $24.48 \%$ & $28.50 \%$ & $28.24 \%$ \\
\hline Mother & $18.27 \%$ & $23.73 \%$ & $20.68 \%$ & $18.80 \%$ & $20.37 \%$ \\
\hline Uterus & $5.92 \%$ & $4.24 \%$ & $6.07 \%$ & $5.30 \%$ & $5.44 \%$ \\
\hline Intra-partum & $4.58 \%$ & $2.75 \%$ & $8.16 \%$ & $1.30 \%$ & $4.24 \%$ \\
\hline Amniotic fluid & $3.55 \%$ & $0.64 \%$ & $3.61 \%$ & $5.30 \%$ & $3.21 \%$ \\
\hline Umbilical cord & $0.17 \%$ & $2.33 \%$ & $2.09 \%$ & $2.20 \%$ & $1.70 \%$ \\
\hline Unclassified & $15.23 \%$ & $18.17 \%$ & $20.91 \%$ & $16.10 \%$ & $17.60 \%$ \\
\hline
\end{tabular}

Table 2: Maternal causes for the still birth

\begin{tabular}{|c|c|c|c|c|}
\hline Maternal causes & 2016 & 2017 & 2018 & 2019 \\
\hline Diabetes & $18 \%$ & $18 \%$ & $39 \%$ & $21 \%$ \\
\hline Hypertension & $73 \%$ & $72 \%$ & $56 \%$ & $70 \%$ \\
\hline Cholestasis & $3 \%$ & $2 \%$ & $0 \%$ & $3 \%$ \\
\hline Obstructed labour & $5 \%$ & $5 \%$ & $4 \%$ & $3 \%$ \\
\hline HELLP & $1 \%$ & $3 \%$ & $1 \%$ & $3 \%$ \\
\hline
\end{tabular}

*HELLP Hemolysis, Elevated Liver enzymes and Low Platelets

$28.24 \%$ of stillbirths on the average, placental abruption remained the main killer (54\%, $72 \%, 78 \%$ and $76 \%$ ), averaging to about $19.5 \%$ of all the recorded stillbirths. Among the maternal causes, diabetes and hypertension contributed the most (table 2). On the average, diabetes and hypertension were responsible for 5 and $13 \%$ respectively, of all stillbirths. Of other notable causes, ruptured uterus was found to be an important entity taking its toll each year $(6 \%, 4 \%, 6 \%$ and $5.3 \%$ respectively).

\section{DISCUSSION}

In Pakistan, like in many other countries, there have been efforts towards improvement in healthcare. However, since we lack the basic information management, it has been difficult to formulate and implement targeted strategies. Global health statistics showed that around 1.9 million stillbirths occur annually. ${ }^{1}$ Pakistan has been recognized as one of the top 10 countries with the highest stillbirth rate globally. ${ }^{11}$ In order to achieve global Every Newborn Action Plan (ENAP) targets for neonatal deaths and stillbirths, various strategies have been ad- opted but the desirable targets are yet to be achieved. ${ }^{12}$ Important contributory factors to these are a lack of proper definition, classification and recording of data related to stillbirths and hence a need to rectify them has been identified. ${ }^{12}$

In our study the rate of stillbirth was $6.2 \%$ (62/1000 births) which seems to be just tip of ice berg. We noted placental causes on the top and placental abruption was the main killer contributing $19.5 \%$ of stillbirths which is significantly higher than studies conducted in countries like Jordan $(5.7 \%)^{13}$ but is comparable to India(22\%). ${ }^{14}$ Fetal causes contributed in $19.2 \% \%$ cases of stillbirth, among these congenital anomalies were on the top (roughly $2 / 3^{\text {rd }}$ among the fetal causes). On the average fetal congenital abnormalities contributed $14 \%$ to all stillbirths. L0cal figures for lethal congenital abnormalities are variable, Hossain et al described $7 \%{ }^{15}$, Khaskheli et al $16 \%{ }^{16}$ while Aziz et al quoted $24.8 \% .^{17}$ Studies show that for 18 countries with reliable data, congenital abnormalities account for a median of only $7 \cdot 4 \%$ of stillbirths. ${ }^{2}$ Rates at which congenital abnormalities contribute to stillbirths is also affected by availability of good screening and diagnostic facilities as well as whether termination of pregnancies for congenital abnormalities is legal or not. Hence in Ireland, where diagnostic facilities are good but termination is not legal, rates as high as $21 \%$ are quoted, while in Qatar, where termination is allowed upto $5^{\text {th }}$ month, these contribute to $11.4 \%$ of stillbirths. ${ }^{2}$ In our country, there is limited availability of adequate diagnostic facilities whereas, although gestational age limit for termination of pregnancy for Thalassemia Major is defined, that for congenital abnormalities is neither defined nor monitored.

Maternal causes were responsible for $1 / 5^{\text {th }}$ cases of stillbirths $(20.37 \%)$. Similar figures have been quoted by Afshan et al (19\%). ${ }^{18}$ Studies from India ${ }^{14}$ and Jordan ${ }^{13}$ also describe similar figures. Among the studied maternal causes, diabetes and hypertension were contributing the most $(5 \%$ and $13 \%$ respectively). Global estimates for contribution of these disorders to stillbirths is approximately $10 \%{ }^{2}$ Due to low uptake of APLS testing due to cost implications, the category of stillbirths due to APLS in mother was limited to already diagnosed cases, and hence, it is possible that this lack of testing might have led to overrepresentation of hypertension as contributory cause. This situation is clearly influenced by increasing maternal age (in women with high parity) and global epidemic of obesity. For the modifiable risk factors, improved healthcare prior to conception, early detection and timely management of both pre-pregnancy medical conditions as well as pregnancy associated medical disorders can avert many untoward effects including stillbirths.

An important area that deserves specific mention is intrapartum stillbirth, which clearly indicate failure of healthcare system to provide emergency obstetric care. Not only there is loss of fetus that has otherwise successfully reached the end of its intrauterine life but may be accompanied by devastating 
consequences of uterine rupture when the labour is obstructed. In our study, there were $4.2 \%$ intrapartum stillbirths with an additional $5.4 \%$ on the average accompanied by uterine rupture. The proportion of stillbirths that are intrapartum varies from $10.0 \%$ (range 5.5-18.4\%) in developed countries to $59.3 \%$ (range $32 \cdot 0-84.0 \%$ ) in south Asia. ${ }^{2}$ Our data matches developed regions probably because it is basically tertiary care hospital based with no inclusion of general community based data, where standard of care is variable and is more often compromised. National surveys quote much higher incidence of intrapartum stillbirths (roughly 40\%)..$^{18}$ Maintaining better standards of intrapartum care can not only prevent fetal but also maternal mortality and morbidity, and for achieving this, simple use of partogram can make a major difference.

The aim of adopting any classification system for stillbirths is to reduce the number of unexplained stillbirths. In our study, stillbirths remained unclassified in $17.6 \%$ of cases. Aziz et al mentioned 19\% as unexplained in a hospital based study, while national surveys quote a much higher figure of $54 \%$ as most of these are out of hospital deliveries without proper antepartum or intrapartum care..$^{18}$ The range of variability is also high in studies from other countries, depending on diagnostic ability and reporting $(3.8-57.4 \%){ }^{19}$

To start with any strategy and policymaking, it is paramount to identify areas where even small interventions can be expected to yield major effect. In light of our study results, it is recommended that antenatal care is needed to be strengthened in field of diagnosis, along with intrapartum care. This in turn is expected to decrease the overall burden on healthcare system coping not only with stillbirths but also with long term disabilities of those mothers and babies who narrowly escape death. The data needs to be recorded and monitored at national level so that awareness, acceptability and access to standardized healthcare can be improved.

\section{CONCLUSION}

The study concluded that overall placental causes were more pre-dominant cause for still births followed by fetal causes. Placental abruption, and congenital anomaly were the most common sub-causes among their categories of placental and fetal causes respectively. Our study showed that even in low resource settings and with limitations, reliable data can be generated through ReCoDe classification and found it a good method for recording stillbirths.

\section{REFERENCES}

1. Hug L, You D, Blencowe H, Mishra A, Wang Z, Fix MJ, et al. Global, regional, and national estimates and trends in stillbirths from 2000 to 2019: a systematic assessment. Lancet 2021; 398(10302):772-85.

2. Lawn JE, Blencowe H, Waiswa P, Amouzou A, Mathers C, Hogan D, et al. Stillbirths: rates, risk factors, and acceleration towards 2030. Lancet 2016; 387(10018):587-603.

3. Stillbirth estimates. 2020. Available from: URL: https://data.unicef.org/topic/child-survival/stillbirths/.

4. Aziz A, Saleem S, Nolen TL, Pradhan NA, McClure EM, Jessani S, et al. Why are the Pakistani maternal, fetal and newborn outcomes so poor compared to other low and middle-income countries? Reprod Health. 2020; 17(3):190.

5. Waqar F. Why have the MDGs failed? The News. [cited 2017 March 9]. Available from: URL: https://www.thenews. com.pk/print/191044-why-have-themdgs-failed.

6. World Health Organization. Trends in maternal mortality: 1990 to 2013. Estimates by WHO, UNICEF, UNFPA, The World Bank and the United Nations Pop- ulation Division. Geneva: WHO; 2014.

7. World Health Organization. Every newborn action plan, country progress tracking report. Geneva: UNICEF; 2015.

8. Shiffman J, Smith S. Generation of political priority for global health initiatives: a framework and case study of maternal mortality. Lancet. 2007; 370(9595):1370-9.

9. Smith SL, Shiffman J, Kazembe A. Generating political priority for newborn survival in three low-income countries. Glob Pub health. 2014; 9(5):538-54.

10. World Health Organization. International statistical classification of diseases and related health problems. Geneva: WHO; 2004.

11. Hug L, Mishra A, Lee $S$, You D, Moran A, Strong KL, et al. A neglected tragedy The global burden of stillbirths: report of the UN inter-agency group for child mortality estimation. United Nations Children's Fund; 2020.

12. UNICEF TAKU. A Situational Analysis on Stillbirths, Newborn Deaths, and Small \& Sick Newborn Care. 2019.

13. Khader YS, Batieha A, Khader A, Hamadneh S. Stillbirths in Jordan: rate, causes, and preventability. J Matern Fetal Neonatal Med. 2020; 33(8):1307-14.

14. Korde VN, Gaikwad PR. Causes of stillbirth. J Obstet Gynecol India. 2008; 58(4):314-8.

15. Hossain N, Khan N, Khan NH. Obstetric causes of stillbirth at low socioeconomic settings. J Pak Med Assoc. 2009; 59(11):744-7.

16. Khaskheli M, Baloch S, Khushk IA, Shah SS. Pattern of fetal deaths at a university hospital of Sindh. J Ayub Med Coll Abbottabad. 2007; 19(2):32-4.

17. Aziz S NM, Akhter S, Shahid R. Frequency of Stillbirths at MCH Centre FGPC Islamabad. J Soc Obstet Gynaecol Pak. 2018; 8(1):9-14.

18. Afshan K, Narjis G, Qayyum M. Risk factors and causes of stillbirths among pregnant women in Pakistan. Afr Health 
Sci. 2019; 19(1):1507-16.

19. Aminu M, Unkels R, Mdegela M, Utz B, Adaji S, van den Broek N. Causes of and factors associated with stillbirth in

Gynaecol. 2014; 121(4):141-53. systematic literature review. Int J Obstet

\section{Author's Contribution}

$\mathrm{NH}$ conceived and designed the research study and also analyzed the data. LZ and MS drafted, reviewed and interpreted the data. Authors agree to be accountable for all aspects of the work in ensuring that questions related to the accuracy or integrity of any part of the work are appropriately : investigated and resolved.

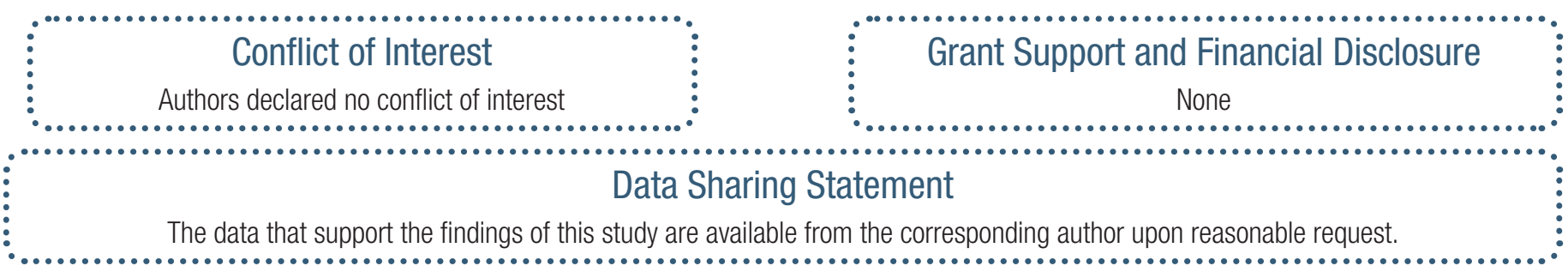

